\title{
A panel of DNA methylation signature from peripheral blood may predict colorectal cancer susceptibility
}

Justina Ucheojor Onwuka, Dapeng Li, Yupeng Liu, Hao Huang, Jing Xu, Ying Liu, Yuanyuan Zhang and Yashuang Zhao* (1)

\begin{abstract}
Background: Differential DNA methylation panel derived from peripheral blood could serve as biomarkers of CRC susceptibility. However, most of the previous studies utilized post-diagnostic blood DNA which may be markers of disease rather than susceptibility. In addition, only a few studies have evaluated the predictive potential of differential DNA methylation in CRC in a prospective cohort and on a genome-wide basis. The aim of this study was to identify a potential panel of DNA methylation biomarkers in peripheral blood that is associated with CRC risk and therefore serve as epigenetic biomarkers of disease susceptibility.

Methods: DNA methylation profile of a nested case-control study with 166 CRC and 424 healthy normal subjects were obtained from the Gene Expression Omnibus (GEO) database. The differentially methylated markers were identified by moderated t-statistics. The DNA methylation panel was constructed by stepwise logistic regression and the least absolute shrinkage and selection operator in the training dataset. A methylation risk score (MRS) model was constructed and the association between MRS and CRC risk assessed.
\end{abstract}

Results: We identified 48 differentially methylated CpGs sites, of which 33 were hypomethylated. Of these, sixteenCPG based MRS that was associated with $C R C$ risk (OR $=2.68,95 \% \mathrm{Cl}: 2.13,3.38, P<0.0001)$ was constructed. This association is confirmed in the testing dataset $(\mathrm{OR}=2.02,95 \% \mathrm{Cl}: 1.48,2.74, P<0.0001)$ and persisted in both males and females, younger and older subjects, short and long time-to-diagnosis. The MRS also predicted CRC with AUC 0.82 (95\% Cl: 0.76, 0.88), indicating high accuracy.

Conclusions: Our study has identified a novel DNA methylation panel that is associated with CRC and could, if validated be useful for the prediction of CRC risk in the future.

Keywords: Colorectal cancer, DNA methylation, Methylation risk score, Peripheral blood

\section{Background}

Colorectal cancer $(\mathrm{CRC})$ poses a great public health concern globally. It is the third most common cancer diagnosed among men and the second most common among women and was responsible for an estimated 1.8

\footnotetext{
*Correspondence: zhao_yashuang@263.net

Department of Epidemiology, Public Health College, Harbin Medical University, 157 Baojian Street, Nangang District, Harbin 150081, Heilongjiang Province, People's Republic of China
}

million new cases and 881,000 deaths in 2018 [1]. In the United States of America, CRC is the third most common cancer diagnosed with about 140,250 new cases and 50,630 deaths in 2017 [2]. In addition to environmental factors, there is proven evidence that CRC results from the accumulation of genetic and epigenetic changes, which changes colonic epithelial cells into adenocarcinoma cells [3].

(c) The Author(s). 2020 Open Access This article is licensed under a Creative Commons Attribution 4.0 International License, which permits use, sharing, adaptation, distribution and reproduction in any medium or format, as long as you give appropriate credit to the original author(s) and the source, provide a link to the Creative Commons licence, and indicate if changes were made. The images or other third party material in this article are included in the article's Creative Commons licence, unless indicated otherwise in a credit line to the material. If material is not included in the article's Creative Commons licence and your intended use is not permitted by statutory regulation or exceeds the permitted use, you will need to obtain permission directly from the copyright holder. To view a copy of this licence, visit http://creativecommons.org/licenses/by/4.0/ The Creative Commons Public Domain Dedication waiver (http://creativecommons.org/publicdomain/zero/1.0/) applies to the data made available in this article, unless otherwise stated in a credit line to the data. 
Epigenetic alterations such as DNA methylation has been associated with many human diseases including cancer and have also been reported to occur early in the development of colorectal tumors [3] by playing a role in gene expression and genomic stability. DNA methylation markers show great potential in the detection and diagnosis of cancer [4] and a panel of differential DNA methylation could be a possible biomarker of CRC susceptibility.

Peripheral blood is an easily accessible source of genomic DNA that can be used to estimate DNA methylation profiles and could serve as useful non-invasive and informative biomarkers for cancer risk [5]. Several studies have investigated peripheral blood DNA methylation biomarkers in different cancer types including head and neck, urothelial, breast, lung, bladder, gastric cancer, prostate, and ovarian cancers [6-16]. Some epidemiologic studies have assessed peripheral blood DNA methylation biomarkers in CRC. However, most of the studies used post-diagnostic blood DNA which may imply that DNA methylation alterations could be an early response of the hematologic system to the presence of CRC cells $[17,18]$. The few studies that utilized prediagnostic DNA focused on genomic methylation of leukocyte DNA [19, 20] while other studies involved candidate genes [21-23] and methylation at repetitive elements [24]. There are few genome-wide DNA methylation studies that have evaluated the association of prediagnostic peripheral blood DNA with CRC risk.

In order to identify a potential panel of DNA methylation biomarkers in peripheral blood that are associated with CRC risk and therefore serve as epigenetic biomarkers of disease susceptibility, we performed an epigenome-wide analysis of a nested case-control study using peripheral blood Illumina HumanMethylation450 bead-array DNA methylation data. We repurposed data previously analysed by Cordero et al who focused on probes associated with genes encoding for miRNAs [25]. We analysed the data using two methods including epigenome-wide methylation profiling to identify differentially methylated CpGs as well as a machine learning algorithm to construct a sixteen-CpG based methylation risk score predictive of $\mathrm{CRC}$ risk.

\section{Methods}

\section{Data source}

The Illumina Human Methylation 450 Beadchip data of the Italian arm of the European Prospective Investigation into Cancer and Nutrition (EPIC-Italy) were obtained from Gene Expression Omnibus (GEO) with the accession number GSE51032. The EPIC is a multicenter prospective study aimed at investigating the complex relationships between nutrition and various lifestyle factors and the etiology of cancer and other chronic diseases
[26]. The EPIC-Italy cohort that was produced in Turin, Italy, is a sub-cohort that comprised of 46,857 volunteers, recruited from five different centers within Italy (Varese, Turin, Florence, Naples and Ragusa) with standardized lifestyle and personal history questionnaires, anthropometric data as well as blood samples collected for DNA extraction. At the last follow-up (2010), 424 participants remained cancer-free, 166 had developed primary colorectal cancer. We extracted the data containing the DNA methylation status of $485,512 \mathrm{CpG}$ sites in the 166 participants who had developed primary colorectal cancer and the 424 matched cancer-free participants.

\section{DNA methylation profiling in CRC and healthy normal subjects}

The differential methylation analysis was conducted using the workflow by Maksimovic et al. [27]. Briefly, we pre-processed and normalized the data using $\mathrm{R}$ package minfi [28]. The quality control, pre-filtering were conducted with the minfi package and the Functional Normalization (FunNorm) function was used for normalization [28, 29]. Quality control was performed and probes with detection $P$-value $>0.01$ in at least one sample were filtered out. After normalization, all probes containing single nucleotide polymorphism (SNPs) and probes mapped to sex chromosomes were filtered out to prevent bias due to unknown genetic background and mixed gender of samples, respectively. Cross-reactive probes, which refer to probes that have shown to map to several positions in the genome [30] were also filtered out. After normalization and quality control, the probes yielded were used for further analysis.

\section{Hierarchical clustering}

We conducted Hierarchical clustering using complete linkage with a Euclidian distance in the R package pheatmap [31].

\section{Functional analysis}

In order to examine main biological functions that were controlled by DNA methylation, we used DMPs (differentially methylated positions) for Gene ontology (GO) analyses and Kyoto Encyclopedia of Genes and Genomes (KEGG) based on the gometh function in the R package missMethyl [32].

\section{Selection of differentially methylated markers for risk model}

The methylation level of all the probes was indicated as beta $(\beta)$ values, which is the proportion of the methylated probe intensity to the total probe intensity (sum of methylated and unmethylated probe intensities plus constant $\alpha$, where $\alpha=100$ ). The beta values for CRC and 
healthy normal subjects were log-transformed to obtain the M-values and used for further analysis, with the beta values used for visualization while the $\mathrm{M}$-values were used for statistical analysis which is in conformity with $\mathrm{Du}$ et al. [33]. The linear models for microarray data (LIMMA) package was used to identify differentially methylated genes between CRC cases and healthy normal subjects [34]. Moderated t-test and mean methylation value differences (delta $(\Delta)$ beta) were generated and we corrected $P$ values of individual probe for multiple testing using the Benjamini-Hochberg method. A CPG site between CRC and healthy normal subjects was considered significant with a false discovery rate $($ FDR $)<$ 0.05 and $\Delta \beta \geq 5 \%$ and DMPs.

In addition, DMPs were used to build a risk score model. The entire sample of 590 was randomly split into $70 \%$ training and 30\% testing sets using stratified random sampling by case-control status. The stratification was to guarantee an equal distribution of CRC and healthy normal subjects between sets, prevent overfitting the data, and allow for validation of the model. The stepwise logistic regression and least absolute shrinkage and selection operator (LASSO) [35] methods were then applied on the training set to select the best markers for CRC prediction using $R$ packages MASS and glmnet respectively [36, 37]. For the LASSO selection analysis, we used 10-fold cross-validation to identify the tuning parameter and chose the minimum lambda, which is the value of lambda with the smallest mean cross-validated error. Nineteen CpGs were identified by using the stepwise regression method and twenty-two CpGs were identified by using the LASSO analysis. In these two approaches, sixteen overlapping markers were identified between the two methods.

\section{Construction of methylation risk score}

Logistics regression models were fitted on the training dataset using these sixteen markers and MRS for each patient was calculated. The calculation was carried out by multiplying the methylation level for each $\mathrm{CpG}$ site with the corresponding regression coefficient and summed over all CpG sites as follows:

$$
\operatorname{MRS}=\beta_{1} x_{1}+\beta_{2} x_{2}+\beta_{3} x_{3}+\ldots \ldots \ldots \ldots+\beta_{k} x_{k}
$$

Where $\beta$ represents the estimated regression coefficient of the CpG site $\mathrm{k}$ derived from the logistic regression analysis, and $x$ represents the methylation level of the CpG site $\mathrm{k}$.

Furthermore, we determined whether our findings could be validated in the testing dataset. The MRS was constructed on the training set and validated on the testing set by fitting a logistic regression model to determine the association of the MRS with CRC, with the MRS added into the model as a continuous variable.

\section{Subgroup analyses}

To assess the robustness of our findings, we determined whether the association between MRS and CRC risk differed by gender, age, and time-to-diagnosis by conducting subgroup analyses according to these variables both in the training and testing datasets. We took advantage of the prospective design of this study and explored the effect of time-to-diagnosis. We categorized the CRC subjects into short (less than 6 years) and long (above 6 years) time-to-diagnosis using the median as a cut-off. In addition, we conducted a case-only analysis and assessed whether methylation levels of the CpGs were correlated with time-to-diagnosis (the time interval between blood draw and diagnosis of CRC).

\section{External validation in TCGA tissues}

In order to validate the predictive performance of the sixteen-CpG panel MRS in an independent dataset, we analysed the CRC data in TCGA (The Cancer Genome Atlas) dataset. The level 3 DNA methylation data detected by HumanMethylation 450 in colon cancer and rectal cancer were downloaded from UCSC Xena (https://xena.ucsc.edu/). We constructed a univariate logistic regression model using the $13-\mathrm{CpGs}$ differentially methylated in TCGA.

\section{Statistical analysis}

The distribution of the demographic characteristics in the study group was compared between CRC and healthy normal subjects using Chi-square and KruskalWallis tests for categorical and continuous data respectively. To estimate the difference in methylation level between CRC and healthy normal, two-sample t-tests (moderated t-tests) with Bonferroni correction was performed for each CpG. Univariate and multivariate logistic regression were used to estimate odds ratios (ORs) and corresponding 95\% confidence intervals (CI) for DNA methylation and MRS between CRC and healthy normal subjects, as well as subgroup analysis. The ROC curves were plotted with $\mathrm{R}$ package pROC version 1.16 .1 [38], to estimate the discriminatory power of the MRS. The area under the ROC curve (AUC) was calculated and the DeLong method was used to calculate the 95\% confident interval (CI) for AUC. The Correlation was performed using Pearson's method. The significance level used for all tests was two-tailed $P<0.05$. All statistical analyses were carried out using $\mathrm{R}$ language software version 3.5.1 (https://cran.r-project.org/bin/windows/ base/old/3.5.1/). 


\section{Results}

Identification of differentially methylated markers

The workflow showing the step-by-step procedure for this analysis and the demographic characteristics of participants are presented in Fig. 1 and Table 1 respectively. We analysed the microarray methylation profile of 166 (87 males and 79 females) CRC and 424 (84 males and 340 females) healthy normal subjects. The average age of CRC subjects was 55 years old whereas, the normal subjects had a mean age of 53. CRC and healthy normal subjects were statistically significantly different with respect to gender but did not differ with respect to age. We adjusted for age and gender in our models. The average time-to-diagnosis for cases was 6.2 years (range $=0-14.3$ ). The Illumina Human Methylation 450 Beadchip contained the DNA methylation status of 485 , $512 \mathrm{CpG}$ sites. Pre-processing and quality control were performed and the poor performing probes were filtered out. A total of 399,934 CpG sites (Additional file 1: Figure S1) were yielded, and their methylation data were used for further analysis. A total of 49,299 CpGs (corresponding with 11, 786 unique genes) were differentially methylated $(F D R<0.05)$ between the CRC and healthy normal subjects.

Gene Ontology (GO) terms and KEGG pathway enrichment analysis for genes associated with the 49,299 differentially methylated CpGs were performed. The GO analysis showed the molecular functions, cellular components, and biological functions of differentially methylated genes under the criterion $F D R<0.05$ (Additional file 2: Table S1). In the KEGG pathway genes showed enrichments in the metabolic pathway $(F D R=1.19 \mathrm{e}-03), \quad$ cancer - pathways $(F D R=6.58 \mathrm{e}-03)$, human papillomavirus infection $(F D R=1.61 \mathrm{e}-02)$, Rap1 signaling pathway $(F D R=4.36 \mathrm{e}-04)$ and Axon guidance $(F D R=2.12 \mathrm{e}-03)$ (Additional file 3: Table S2).

Of the 49,299 CpGs differentially methylated, 48 CpGs (corresponding with 29 unique genes) which had absolute mean $\beta$-value difference $(|\Delta \beta| \geq 0.05)$ were selected and denoted DMPs (Additional file 4: Table S3). Among the DMPs, a total of $15 \mathrm{CpGs}$ (corresponding with 8 unique genes) were hypermethylated and $33 \mathrm{CpGs}$ (corresponding with 21 unique genes) were hypomethylated. Hierarchical clustering was implemented to determine whether the identified DMPs could distinguish CRC from healthy normal subjects. The results showed a significant difference in methylation between CRC and healthy normal subjects (Fig. 2).

\section{Methylation risk score construction}

The entire sample of 590 was randomly split into training (117 CRC subjects and 297 healthy normal subjects)

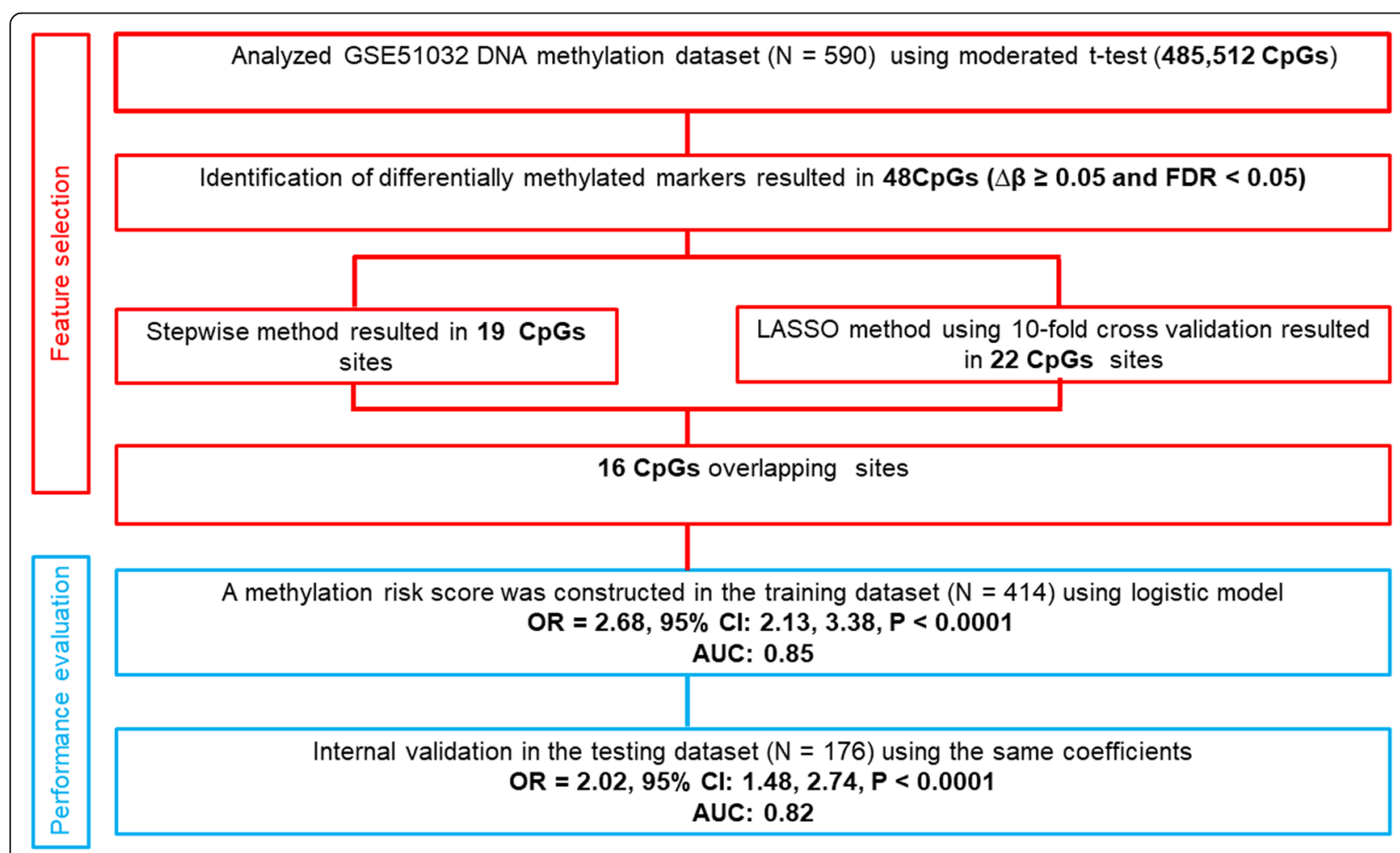

Fig. 1 Overall workflow of the step-by-step analyses process of this study 
Table 1 Characteristics of Training and Testing Dataset of Nested Case Control Study Based on EPIC-Italy Cohort

\begin{tabular}{|c|c|c|c|c|c|c|}
\hline \multirow[t]{2}{*}{ Characteristics } & \multicolumn{2}{|c|}{ Entire Dataset } & \multicolumn{2}{|c|}{ Training Dataset } & \multicolumn{2}{|c|}{ Testing Dataset } \\
\hline & Cases & Control & Cases & Control & Cases & Control \\
\hline Total & 166 & 424 & 117 & 297 & 49 & 127 \\
\hline Age, Mean (SD) & $55.07(6.73)$ & $53.23(7.19)$ & $55.94(6.73)$ & $53.08(7.20)$ & $55.25(6.62)$ & $53.56(7.20)$ \\
\hline$<60$ & $128(26.9)$ & $348(73.1)$ & $89(26.4)$ & $245(73.4)$ & $10(27.5)$ & $103(72.5)$ \\
\hline$\geq 60$ & $38(33.3)$ & $76(66.7)$ & $28(35.0)$ & $52(65.0)$ & $39(29.4)$ & $24(70.6)$ \\
\hline \multicolumn{7}{|l|}{ Gender } \\
\hline Male & $87(52.4)$ & $84(19.8)$ & $55(47.4)$ & $61(52.6)$ & $32(58.2)$ & $23(41.8)$ \\
\hline Female & 79 (47.6) & $340(80.2)$ & $62(20.8)$ & $236(79.2)$ & $17(14.0)$ & $104(86.2)$ \\
\hline \multicolumn{7}{|c|}{ Time-to-diagnosis (years) } \\
\hline$<6$ & $80(48.2)$ & NA & $56(47.9)$ & NA & $24(49.0)$ & NA \\
\hline$\geq 6$ & $86(51.8)$ & NA & $61(52.1)$ & NA & $25(51.0)$ & NA \\
\hline
\end{tabular}

Abbreviations: NA Not applicable, SD Standard deviation

and testing (49 CRC subjects and 127 healthy normal subjects) sets (Table 1). Differentially methylated markers associated with CRC risk were screened on the training dataset using LASSO selection and stepwise logistic regression analysis. The sixteen markers mapped to nine genes including LGR6, PTPN12, PPFIA3, LOC399959, PCDHGA1, RNF39, ESYT3, MRGPRG, and ATHL1 overlapping between the two methods were selected (Additional file 5: Figure S2). The associations of the sixteen individual markers with CRC by univariate and multivariate logistic regression analysis are presented in Additional file 6: Table $S 4$ and Table 2 respectively.

Furthermore, using the sixteen-CpG panel we calculated a methylation risk score (MRS) for each subject on the training dataset using the formula:

$$
\begin{aligned}
\text { MRS }= & (-0.4100 * \operatorname{cg} 06551493) \\
& +(0.4332 * \operatorname{cg} 01419670) \\
& +(0.2895 * \operatorname{cg} 16530981) \\
& +(-0.5172 * \operatorname{cg} 18022036) \\
& +(-0.3915 * \operatorname{cg} 12691488) \\
& +(-0.3246 * \operatorname{cg} 17292758) \\
& +(-0.2886 * \operatorname{cg} 16170495) \\
& +(0.2451 * \operatorname{cg} 11240062) \\
& +(-0.5651 * \operatorname{cg} 21585512) \\
& +(0.3615 * \operatorname{cg} 24702253) \\
& +(-0.2445 * \operatorname{cg} 17187762) \\
& +(-0.3951 * \operatorname{cg} 05983326) \\
& +(-0.5089 * \operatorname{cg} 06825163) \\
& +(-0.2504 * \operatorname{cg} 11885357) \\
& +(-0.2357 * \operatorname{cg} 08829299) \\
& +(-0.3607 * \operatorname{cg} 07044115) .
\end{aligned}
$$

The methylation levels of 4 CpG (cg01419670, cg16530981, cg11240062, cg24702253) sites were hypermethylated, and 12 CpG (cg06551493, cg18022036, cg12691488, cg17292758, cg16170495, cg21585512, cg17187762, cg05983326, cg06825163, cg11885357, cg08829299, cg07044115) sites were hypomethylated.

The MRS (range, -5.59 to 4.35) was significantly higher for CRC subjects than in healthy normal subjects $(P<0.000)$, with a median MRS of 1.68 (IQR, 1.43) in CRC subjects and -0.430 (IQR, 2.89) in healthy normal subjects (Additional file 7: Figure S3a) in the training dataset. The MRS was associated with a 2.68-fold increased risk of CRC (OR $=2.68,95 \%$ CI: 2.13, 3.38, $P<0.0001)$ Table 2 . The MRS showed a good predictive ability for discriminating between CRC and healthy normal subjects (AUC, 0.85; 95\% CI: 0.81, 0.89) Fig. 3a.

\section{Validation of the sixteen-CpG panel MRS for CRC prediction in the testing dataset}

In order to validate the predictive performance of the sixteen-CpG panel MRS for the prediction of CRC risk, the predictive model was applied to the testing dataset. The MRS (range, -5.73 to 3.89 ) was also significantly higher for CRC subjects than in healthy normal subjects $(P<0.0001)$, with median MRS of 1.83 (IQR, 1.80) in CRC subjects and -0.45 (IQR, 2.64) in healthy normal subjects (Additional file 7: Figure S3b). Consistent with the training dataset, the MRS was associated with a 2.02-fold increased risk of CRC (OR $=2.02,95 \% \mathrm{CI}: 1.48$, $2.74, P<0.0001)$ Table 2 . Similar to the training dataset, the MRS showed a good predictive ability for discriminating between CRC and healthy normal subjects (AUC, 0.82; 95\% C: 0.76, 0.88) Fig. 3b.

\section{Subgroup analysis for the association between MRS and $\mathrm{CRC}$ risk}

When the study subjects were stratified according to gender, age and time-to-diagnosis, the MRS still 


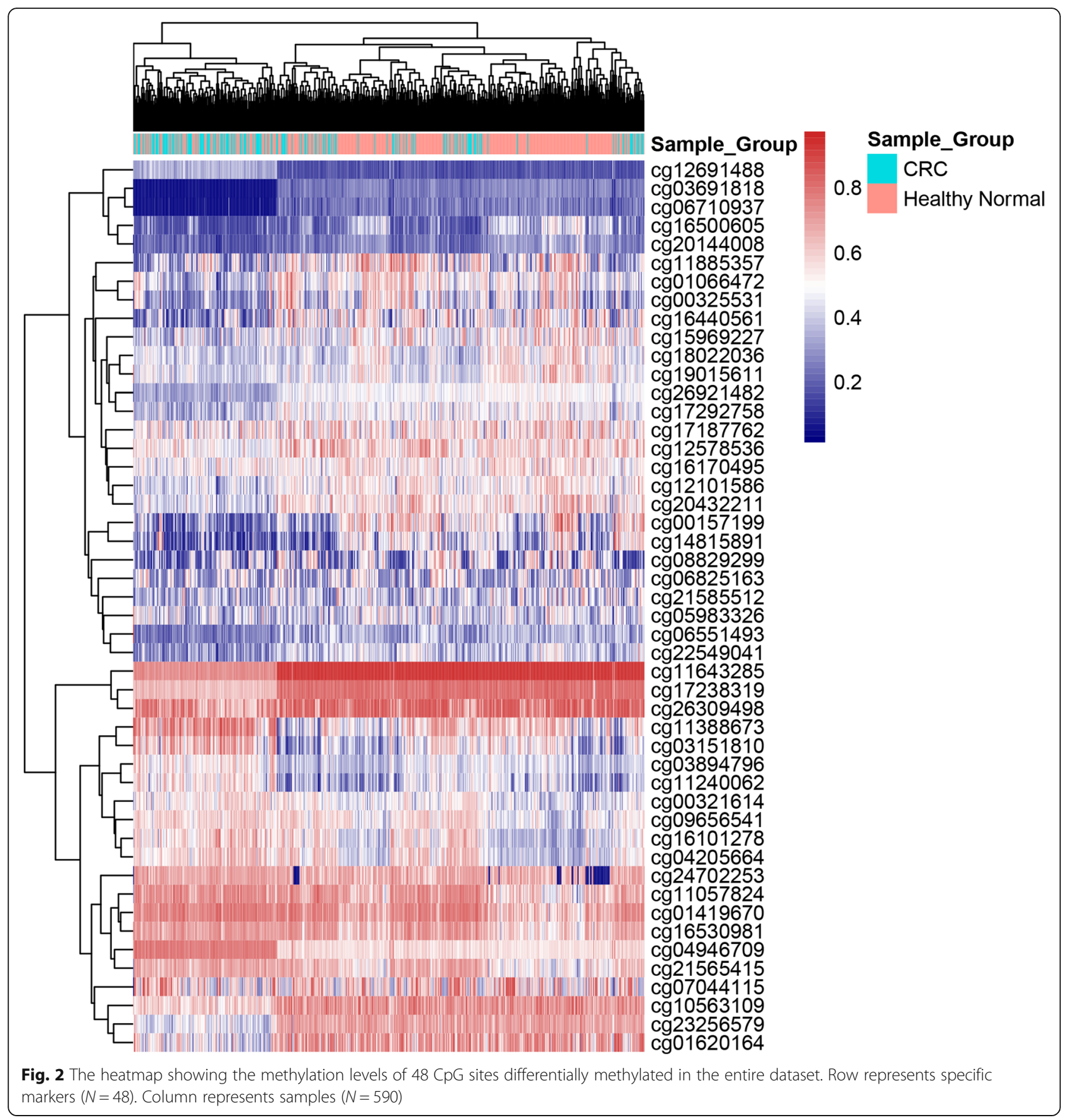

demonstrated an increased risk of CRC among both male and female subjects, younger $(<60$ years) and the older ( $\geq 60$ years) subjects as well as short and long time to diagnosis in the training and testing datasets (Table 3 ). Also, the case-only analysis demonstrated no correlation between methylation levels time-to-diagnosis, with only one CpG showing small negative correlation (Additional file 8: Table S5).
Independent validation of the sixteen-CpG panel MRS for CRC prediction in TCGA dataset

We used TCGA dataset of $391 \mathrm{CRC}$ and 45 controls for independent validation of our sixteen-CpG panel MRS. Only thirteen CpGs of the panel were differentially methylated in the TCGA dataset. The beta values of the thirteen CpGs were extracted and univariate logistic regression models were constructed (Additional file 9: Table S6). We identified nine CpGs (cg06551493, 
Table 2 Multivariate Analysis on the Associations of DNA Methylation Marker, MRS and Risk of CRC of Nested Case Control Study Based on EPIC-Italy Cohort

\begin{tabular}{|c|c|c|c|c|c|c|c|c|c|c|}
\hline \multirow[t]{2}{*}{ CpG ID } & \multirow{2}{*}{$\begin{array}{l}\text { Gene } \\
\text { Name }\end{array}$} & \multicolumn{3}{|c|}{ Entire Dataset } & \multicolumn{3}{|c|}{ Training Dataset } & \multicolumn{3}{|c|}{ Testing Dataset } \\
\hline & & $\overline{O R}$ & $95 \% \mathrm{Cl}$ & $P$-value & $\overline{O R}$ & $95 \% \mathrm{Cl}$ & $P$-value & $\overline{O R}$ & $95 \% \mathrm{Cl}$ & $P$-value \\
\hline cg06551493 & PTPN12 & 0.62 & $0.49,0.78$ & $6.58 \mathrm{e}-05$ & 0.71 & $0.54,0.91$ & 0.009 & 0.42 & $0.25,0.68$ & $7.18 \mathrm{e}-04$ \\
\hline cg01419670 & NA & 2.12 & $1.62,2.85$ & $1.47 e-07$ & 2.36 & $1.71,3.36$ & $6.16 e-07$ & 1.62 & $1.00,2.82$ & 0.06 \\
\hline cg16530981 & NA & 1.96 & $1.52,2.57$ & $5.01 \mathrm{e}-07$ & 2.15 & $1.60,2.98$ & $1.29 \mathrm{e}-06$ & 1.53 & $0.99,2.57$ & 0.08 \\
\hline cg18022036 & NA & 0.56 & $0.45,0.70$ & $5.47 e-07$ & 0.54 & $0.41,0.69$ & $3.97 e-06$ & 0.67 & $0.43,1.03$ & 0.07 \\
\hline $\operatorname{cg} 12691488$ & NA & 0.73 & $0.47,1.10$ & 0.14 & 0.67 & $0.40,1.08$ & 0.11 & 0.84 & $0.34,1.95$ & 0.70 \\
\hline cg17292758 & PPFIA3 & 0.79 & $0.64,0.98$ & 0.04 & 0.79 & $0.61,1.02$ & 0.07 & 0.79 & $0.51,1.20$ & 0.27 \\
\hline cg16170495 & RNF39 & 0.66 & $0.54,0.80$ & $3.17 e-05$ & 0.68 & $0.54,0.85$ & 0.001 & 0.62 & $0.41,0.90$ & 0.01 \\
\hline cg11240062 & NA & 1.25 & $1.00,1.57$ & 0.06 & 1.30 & $1.00,1.69$ & 0.05 & 1.11 & $0.70,1.78$ & 0.67 \\
\hline cg21585512 & LOC399959 & 0.68 & $0.55,0.83$ & $1.61 \mathrm{e}-04$ & 0.58 & $0.45,0.74$ & $1.64 \mathrm{e}-05$ & 0.94 & $0.64,1.38$ & 0.75 \\
\hline cg24702253 & MRGPRG & 1.74 & $1.28,2.58$ & 0.002 & 1.78 & $1.24,2.81$ & 0.005 & 0.74 & $1.01,4.58$ & 0.11 \\
\hline cg17187762 & NA & 0.76 & $0.63,0.93$ & 0.006 & 0.78 & $0.62,0.97$ & 0.03 & 0.66 & $0.44,0.98$ & 0.04 \\
\hline cg05983326 & PCDHGA1 & 0.69 & $0.57,0.84$ & $2.68 \mathrm{e}-04$ & 0.73 & $0.57,0.91$ & 0.007 & 0.57 & $0.38,0.84$ & 0.006 \\
\hline cg06825163 & LGR6 & 0.70 & $0.57,0.86$ & $5.47 \mathrm{e}-04$ & 0.67 & $0.52,0.84$ & $8.11 \mathrm{e}-04$ & 0.82 & $0.56,1.21$ & 0.34 \\
\hline $\operatorname{cg} 11885357$ & ESYT3 & 0.89 & $0.73,1.08$ & 0.23 & 0.83 & $0.65,1.04$ & 0.11 & 1.07 & $0.72,1.59$ & 0.74 \\
\hline cg08829299 & ATHL1 & 0.86 & $0.70,1.04$ & 0.13 & 0.84 & $0.66,1.05$ & 0.13 & 0.93 & $0.63,1.37$ & 0.69 \\
\hline cg07044115 & NA & 0.77 & $0.63,0.93$ & 0.008 & 0.82 & $0.66,1.03$ & 0.07 & 0.60 & $0.40,0.89$ & 0.01 \\
\hline
\end{tabular}

Abbreviations: $\mathrm{Cl}$ Confidence interval, $C R C$ Colorectal cancer, MRS Methylation risk score, ORs Adjusted for age and gender, $P$ values $<0.05$ are in bold

cg12691488, cg17292758, cg16170495, cg21585512, cg24702253, cg17187762, cg05983326, cg11885357) that were associated with CRC, and the MRS for each sample was calculated. The MRS (range, -4.05 to 2.92 ) was significantly higher for CRC subjects than in controls subjects $(P<0.0001)$, with a median MRS of 0.16 (IQR, 1.59 ) in CRC subjects and - 0.712 (IQR, 0.95) in controls (Additional file 10: Figure S4). The MRS was associated 1.96-fold increased risk in CRC (OR $=2.06,95 \% \mathrm{CI}: 1.55$, 2.78, $P<1.08 \mathrm{e}-06$ ) (Additional file 9: Table S6). The
MRS showed a good predictive ability for discriminating between CRC and control subjects (AUC, 0.73; 95\% CI: 0.66-0.79) Additional file 11: Figure S5.

\section{Discussion}

In this study, we repurposed a microarray peripheral blood DNA methylation data of CRC and healthy normal subjects obtained from the GEO database. First, we identified differentially methylated CpGs between CRC and healthy normal subjects for CRC-specific
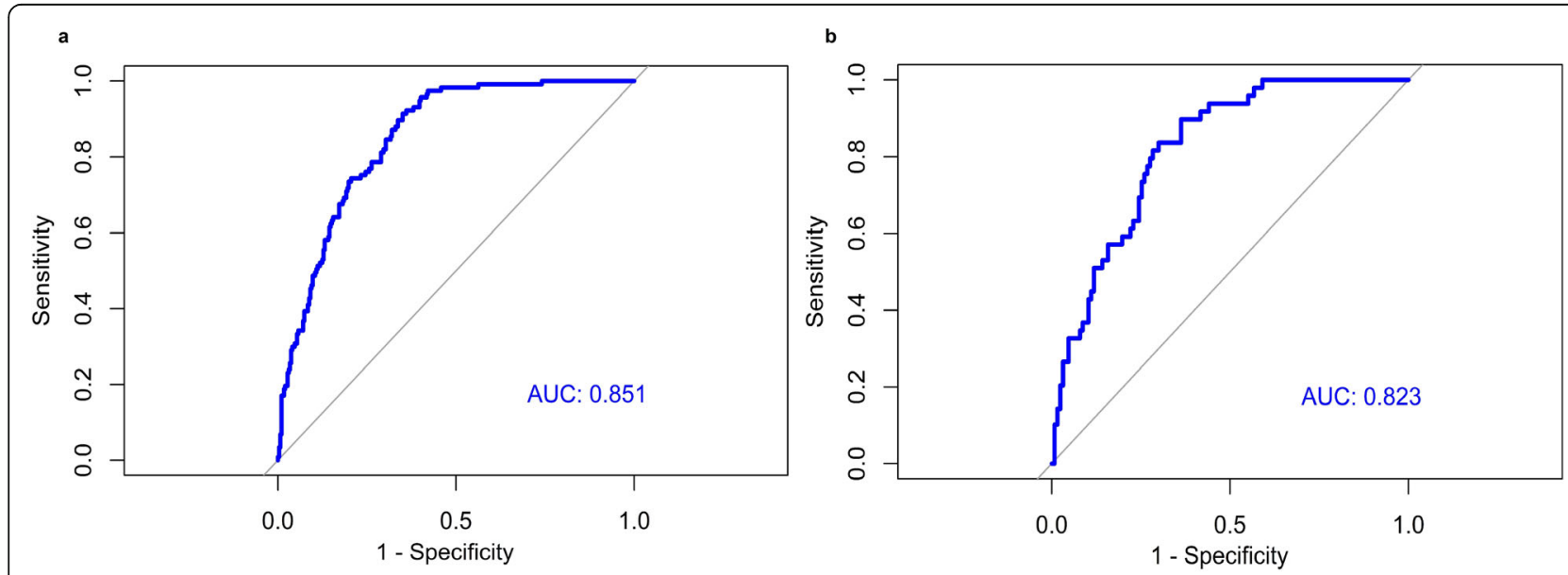

Fig. 3 Classification performance of methylation risk score (MRS) for CRC risk (a) Receiver operating characteristic (ROC) curve for methylation risk score (MRS) prediction of CRC, with area-under the curve (AUC) of 0.85 (95\% Cl: 0.82-0.88) on training dataset. b Receiver operating characteristic (ROC) curve for methylation risk score (MRS) prediction of CRC, with area-under the curve (AUC) 0.82 (95\% Cl: 0.76-0.88) on testing dataset 
Table 3 Associations of MRS and Risk of CRC According to Age, Gender and Time-To-Diagnosis of Nested Case Control Study Based on EPIC-Italy Cohort

\begin{tabular}{|c|c|c|c|c|c|c|c|c|c|}
\hline \multirow[t]{2}{*}{ Characteristics } & \multicolumn{3}{|c|}{ Entire Dataset } & \multicolumn{3}{|c|}{ Training Dataset } & \multicolumn{3}{|c|}{ Testing Dataset } \\
\hline & $\mathrm{OR}$ & $95 \% \mathrm{Cl}$ & $P$-value & $\mathrm{OR}$ & $95 \% \mathrm{Cl}$ & $P$-value & $\mathrm{OR}$ & $95 \% \mathrm{Cl}$ & $P$-value \\
\hline \multicolumn{10}{|l|}{ Age } \\
\hline$<60$ & 2.35 & $1.95,2.90$ & $<0.0001$ & 2.62 & $2.06,3.44$ & $<0.0001$ & 1.97 & $1.44,2.84$ & $<0.0001$ \\
\hline$\geq 60$ & 2.60 & $1.77,4.17$ & $<0.0001$ & 2.87 & $1.77,5.35$ & 0.0002 & 2.28 & $1.20,5.51$ & 0.03 \\
\hline \multicolumn{10}{|l|}{ Gender } \\
\hline Male & 1.97 & $1.47,2.71$ & $<0.0001$ & 2.10 & $1.44,3.21$ & 0.0002 & 1.91 & $1.20,3.37$ & 0.02 \\
\hline Female & 2.64 & $2.12,3.37$ & $<0.0001$ & 2.96 & $2.26,4.08$ & $<0.0001$ & 2.08 & $1.46,3.20$ & 0.0002 \\
\hline \multicolumn{10}{|l|}{ Time-diagnosis } \\
\hline$<6$ years & 2.21 & $1.80,2.77$ & $<0.0001$ & 2.40 & $1.86,3.20$ & $<0.0001$ & 1.99 & $1.40,3.02$ & 0.0004 \\
\hline$\geq 6$ years & 2.51 & $2.01,3.23$ & $<0.0001$ & 2.88 & $2.17,4.01$ & $<0.0001$ & 1.97 & $1.36,3.05$ & 0.0008 \\
\hline
\end{tabular}

methylation panel. Second, we divided the data into two sets and identified a panel of sixteen CpGs associated with CRC by logistic regression in the training dataset. Third, we constructed a predictive model- MRS, to predict the risk of CRC based on the linear combination of methylation levels of the sixteen CpGs. The MRS was tested first on the training dataset and was associated with the risk of CRC, the prediction evaluation when conducted by ROC analysis attained an AUC of 0.85 . Subgroup analyses demonstrated that these significant associations persisted in both males and females, younger and older subjects as well as long and short time-todiagnosis. The MRS, when validated on the testing dataset attained an AUC of 0.82 indicating that the risk predictive value of the MRS panel is replicable for predicting CRC risk. Our findings show a panel of peripheral blood DNA methylation that is a potential biomarker for CRC susceptibility.

Previous studies have developed multiple gene methylation-based panels to predict an individual's susceptibility to CRC. For example, Liu et al. and Luo et al. both reported DNA methylation-based panels in blood leukocyte that were associated with 6.51-fold (95\% CI, 3.77-11.27) and 1.54-fold (95\% CI: 1.15-2.05) increased risk of CRC respectively and this is similar to our result. However, since both studies involved post-diagnostic DNA samples based on case-control studies, the association detected may have resulted from a response to CRC cells rather than CRC susceptibility.

Although the mechanisms underlying the aberrations in the methylation of peripheral blood DNA among individuals who are susceptible to CRC are not clear, our analysis used pre-diagnostic peripheral blood DNA, which indicates that methylation aberrations in peripheral blood DNA could possibly be a long-term CRC predisposition risk markers or a far early response to CRC cells before cancer could be detected by techniques used before now such as endoscopy and cytology. In addition, there was no correlation between DNA methylation and time-to-diagnosis in case-only analysis, which also supports the suggestion that peripheral blood DNA could be a long-term event.

Contrary to our result, the previous studies that utilized pre-diagnostic blood DNA found no association between pre-diagnostic genomic DNA methylation status and CRC risk $[19,20]$. This difference might be because of the heterogeneous methodology and assays. The two studies evaluated leukocyte genomic DNA methylation levels by liquid chromatography/tandem mass spectrometry, which only considers DNA hypomethylation and not regional hypermethylation that can also contribute to increased risk of CRC.

The presence of specific single nucleotide polymorphisms (SNPs) has also been used to evaluate an individual's risk for CRC both by the candidate and multiple genes (by a method called a genetic risk score (GRS)) as well as genome-wide association study (GWAS). Similar to the associations we found between MRS and CRC risk, GRS based on SNPs have been associated with CRC risk. For example, Cho et al. [39], reported a higher GRS that was associated with CRC (OR, 2.57; 95\% CI, 1.89, 3.49) using thirteen SNPs. In addition, Jung et al. [40] in a casecohort study, demonstrated that participants in the highest quartiles of the genetic risk score had an increased risk of CRC (hazard ratio, 2.65; 95\% CI, 1.43 to 4.91) compared with those in the lowest quartile using seven SNPs. Furthermore, a GWAS study found a SNPs developed polygenic risk score (PRS) that was associated with about 2 -fold increased risk of CRC [41].

In the present study, the methylation-based markers for CRC included LGR6, PTPN12, PPFIA3, LOC399959, PCDHGA1, RNF39, ESYT3, MRGPRG and ATHL1, all of 
which were located in the promoter regions or first introns of nearby genes. There are limited epidemiological reports on the association between these markers and CRC risk. One of the genes, LGR6 (Leucine-Rich Repeat Containing G Protein-Coupled Receptor 6) regulates the phosphoinositide 3-kinase/AKT signaling pathway and plays a tumor-promoting role in CRC development indicating that it might be a potential diagnostic and prognostic biomarker for CRC [42]. Protein tyrosine phosphatase non-receptor type 12 (PTPN12) are signaling molecules that regulate a variety of cellular processes and has been found to be epigenetically regulated in triple-negative breast cancer [43]. They are known to play an important role in cell growth, proliferation, and motility [44] and have been found to function as a suppressor of epithelial cell motility in CRC cells [45]. A study on whole-exome sequencing identified that PTPN12 variant is associated with CRC susceptibility [46]. In addition, the methylation of PTPRF-interacting protein alpha 3(PPFIA3) in serum has shown a potential for the detection of gastric cancer [47].

The pathway analysis demonstrated that metabolic pathways, cancer pathways, human papillomavirus infection, Rap1 signaling pathway, and Axon guidance were associated with CRC. The biological processes involve cellular component organization or biogenesis, and cellular localization. The Rap1 signaling pathway has been implicated in the previous genome-wide profile of colorectal cancer [48] and has been known to play several important roles in tumor cell invasion and metastasis [49]. The pathway and biological processes put together demonstrate that multiple pathways, which were affected by aberrant methylation were involved in CRC tumorigenesis.

In order to validate the MRS, we conducted an independent validation analysis of our results using TCGA dataset for CRC risk prediction. Despite the fact that only 9 CpGs from $16-\mathrm{CpG}$ MRS panel was available in TCGA datasets for calculation of MRS, the MRS was still higher for CRC subjects then controls. It is noteworthy that PTPN12, RNF39, LOC399959, PCDHGA1, and LGR6 are also significantly hypomethylated in CRC tissue compared to normal tissue in the TCGA dataset, suggesting that the changes observed in DNA methylation levels may be clinically important.

To our knowledge, our analysis is the first to assess the potential link between genome-wide DNA methylation in peripheral blood and future risk of CRC. Our analysis has revealed that there is potential in the use of peripheral blood-based DNA methylation profiling for CRC risk prediction. We have shown, with a ROC indicating good performance, an MRS model consisting of sixteen CpG panel that has the ability to differentiate CRC from healthy normal subjects.
One important strength of our study was its prospective design. The utilization of blood samples collected before diagnosis which indicated that the DNA methylation preceded the development of CRC by up to 6 years, enabled us to assess genome-wide measures of DNA methylation as potential biomarkers of risk as compared to measures of DNA methylation in retrospective designs which may have resulted from molecular changes due to carcinogenesis and medication.

A limitation of our study is its lack of replication. To the best of our knowledge, there are currently no other pre-diagnostic blood DNA Illumina Human Methylation 450 data for CRC studies available. However, we used TCGA dataset for external validation and recommend that other prospective cohort studies assess associations between genome-wide DNA methylation and CRC risk.

\section{Conclusion}

Our study has identified a novel DNA methylation panel based on genome-wide analysis that is associated with CRC and suggests that differential peripheral blood DNA methylation panel may be an easily available biomarker for prediction of CRC risk in the future if validated in a prospective cohort. Further studies with larger cohort data will be needed to confirm this pattern.

\section{Supplementary information}

Supplementary information accompanies this paper at https://doi.org/10. 1186/s12885-020-07194-5.

Additional file 1: Figure S1. Volcano plot for differential DNA methylation analysis of all 399,934 CpG sites among 166 CRC and healthy normal subjects. The $x$-axis shows the mean DNA methylation difference (delta beta), while the $y$-axis shows the $-\log 10$ of the $p$ value for each CpG.

Additional file 2: Table S1. Top Gene Ontology Enrichment Analysis of Differentially Methylated Genes of Nested Case Control Study Based on EPIC-Italy Cohort.

Additional file 3: Table S2. KEGG Pathway Enrichment Analysis of Nested Case Control Study Based on EPIC-Italy Cohort.

Additional file 4: Table S3. Differentially Methylated CpGs between Colorectal Cancer and Healthy Normal Subjects of Nested Case Control Study Based on EPIC-Italy Cohort.

Additional file 5: Figure S2. The distribution of the methylation values of the sixteen risk markers in CRC and healthy normal subjects in the training (a) and testing (b) dataset.

Additional file 6: Table S4. Univariate Analysis on the Associations of DNA Methylation Marker, MRS and Risk of CRC of Nested Case Control Study Based on EPIC-Italy Cohort.

Additional file 7: Figure S3. Box-plots of the MRS separately in CRC and healthy normal subjects for training dataset (Fig. S3a) and testing dataset (Fig. S3b).

Additional file 8: Table S5. Correlation between DNA Methylation Marker and Time-To-Diagnosis of Nested Case Control Study Based on EPIC-Italy Cohort. 
Additional file 9: Table S6. Univariate Analysis on the Associations of DNA Methylation Marker, MRS and Risk of CRC TCGA dataset.

Additional file 10: Figure S4. Box-plots of the MRS separately in CRC and control subjects for TCGA dataset.

Additional file 11: Figure S5. Classification performance of methylation risk score (MRS) for CRC risk; Receiver operating characteristic (ROC) curve for methylation risk score (MRS) prediction of CRC, with areaunder the curve (AUC) of 0.73 (95\% Cl: 0.66-0.79) on TCGA dataset.

\section{Abbreviations}

AUC: Area under the ROC curve; Cl: Confidence interval; CRC: Colorectal cancer; DMPs: Differentially methylated probes; ESYT3: Extended Synaptotagmin 3; EPIC: European Prospective Investigation into Cancer and Nutrition; FDR: False discovery rate; GEO: Gene Expression Omnibus; GO: Gene ontology; KEGG: Kyoto Encyclopedia of Genes and Genomes; LASSO: Least absolute shrinkage and selection operator; LIMMA: Linear models for microarray data; LGR6: Leucine Rich Repeat Containing G ProteinCoupled Receptor 6; MRS: Methylation risk score; OR: Odds ratio; PCDH GA1: Protocadherin gamma subfamily A, 1; PPFIA3: PTPRF-interacting protein alpha 3; PTPN12: Protein tyrosine phosphatase non-receptor type 12; ROC: Receiver Operating Characteristic

\section{Acknowledgements}

The authors are grateful to the Gene Expression Omnibus (GEO) database team for the access to the data.

\section{Authors' contributions}

$J \cup O, D L$ and $Y Z$ : designed the study; JUO, DL, HH, YYZ and YL: conducted data acquisition and analysis; JUO, YPL and DL: drafted the manuscript; JUO, $D L$, JX and YZ: revised the manuscript. All authors read and approved the final version for publication.

\section{Funding}

This study was funded by the National Natural Science Foundation of China (81473055). The China Scholarship Council supported JUO (2017BSZ011594). The funders had no role in the design of the study, analysis, interpretation of data and manuscript writing.

\section{Availability of data and materials}

The raw data sets used to support the conclusions of this article are available on NCBI's Gene Expression Omnibus (GEO) through GEO accession number GSE51032. https://www.ncbi.nlm.nih.gov/geo/query/acc.cgi?acc=GSE51032 and The Cancer Genome Atlas (TCGA, https://cancergenome.nih.gov/).

\section{Ethics approval and consent to participate}

The datasets utilized for this study are publicly available and did not require any permissions for use.

\section{Consent for publication}

Not applicable.

\section{Competing interests}

Authors declare that they have no competing interests.

Received: 12 February 2020 Accepted: 19 July 2020

Published online: 25 July 2020

\section{References}

1. Bray F, Ferlay J, Soerjomataram I, Siegel RL, Torre LA, Jemal A. Global cancer statistics 2018: GLOBOCAN estimates of incidence and mortality worldwide for 36 cancers in 185 countries. CA Cancer J Clin. 2018;68(6):394-424.

2. Siegel RL, Miller KD, Jemal A. Cancer statistics, 2018. CA Cancer J Clin. 2018; 68(1):7-30.

3. Grady WM, Carethers JM. Genomic and epigenetic instability in colorectal cancer pathogenesis. Gastroenterology. 2008;135(4):1079-99.

4. Muller HM, Widschwendter M. Methylated DNA as a possible screening marker for neoplastic disease in several body fluids. Expert Rev Mol Diagn. 2003;3(4):443-58.
5. Li L, Choi JY, Lee KM, Sung H, Park SK, Oze I, et al. DNA methylation in peripheral blood: a potential biomarker for cancer molecular epidemiology. J Epidemiol. 2012;22(5):384-94.

6. Barry KH, Moore LE, Liao LM, Huang WY, Andreotti G, Poulin M, et al. Prospective study of DNA methylation at LINE-1 and Alu in peripheral blood and the risk of prostate cancer. Prostate. 2015;75(15):1718-25.

7. Dugue PA, Brinkman MT, Milne RL, Wong EM, FitzGerald LM, Bassett JK, et al. Genome-wide measures of DNA methylation in peripheral blood and the risk of urothelial cell carcinoma: a prospective nested case-control study. Br J Cancer. 2016;115(6):664-73.

8. FitzGerald LM, Naeem H, Makalic E, Schmidt DF, Dowty JG, Joo JE, et al. Genome-wide measures of peripheral blood Dna methylation and prostate Cancer risk in a prospective nested case-control study. Prostate. 2017;77(5): 471-8.

9. Langevin SM, Koestler DC, Christensen BC, Butler RA, Wiencke JK, Nelson $\mathrm{HH}$, et al. Peripheral blood DNA methylation profiles are indicative of head and neck squamous cell carcinoma: an epigenome-wide association study. Epigenetics. 2012;7(3):291-9.

10. Li J, Chen Y, Yu H, Tian J, Yuan F, Fan J, et al. DUSP1 promoter methylation in peripheral blood leukocyte is associated with triple-negative breast cancer risk. Sci Rep. 2017;7:43011.

11. Li L, Zheng H, Huang Y, Huang C, Zhang S, Tian J, et al. DNA methylation signatures and coagulation factors in the peripheral blood leucocytes of epithelial ovarian cancer. Carcinogenesis. 2017;38(8):797-805.

12. Marsit CJ, Koestler DC, Christensen BC, Karagas MR, Houseman EA, Kelsey KT. DNA methylation array analysis identifies profiles of blood-derived DNA methylation associated with bladder cancer. J Clin Oncol. 2011;29(9):1133-9.

13. Sun H, Zhou H, Zhang Y, Chen J, Han X, Huang D, et al. Aberrant methylation of FAT4 and SOX11 in peripheral blood leukocytes and their association with gastric cancer risk. J Cancer. 2018;9(13):2275-83.

14. Wang L, Aakre JA, Jiang R, Marks RS, Wu Y, Chen J, et al. Methylation markers for small cell lung cancer in peripheral blood leukocyte DNA. J Thorac Oncol. 2010;5(6):778-85.

15. Xu X, Gammon MD, Hernandez-Vargas H, Herceg Z, Wetmur JG, Teitelbaum $S L$, et al. DNA methylation in peripheral blood measured by LUMA is associated with breast cancer in a population-based study. FASEB J. 2012; 26(6):2657-66.

16. Zhang $Y$, Zhou $H$, Sun $H$, Chen J, Huang D, Han $X$, et al. Association of peripheral blood leukocyte KIBRA methylation with gastric cancer risk: a case-control study. Cancer Med. 2018;7(6):2682-90.

17. Liu Y, Wang Y, Hu F, Sun H, Zhang Z, Wang $X$, et al. Multiple gene-specific DNA methylation in blood leukocytes and colorectal cancer risk: a casecontrol study in China. Oncotarget. 2017;8(37):61239-52.

18. Luo X, Huang R, Sun H, Liu Y, Bi H, Li J, et al. Methylation of a panel of genes in peripheral blood leukocytes is associated with colorectal cancer. Sci Rep. 2016;6:29922.

19. Huang WY, Su LJ, Hayes RB, Moore LE, Katki HA, Berndt SI, et al. Prospective study of genomic hypomethylation of leukocyte DNA and colorectal cancer risk. Cancer Epidemiol Biomark Prev. 2012;21(11):2014-21.

20. Nan H, Giovannucci EL, Wu K, Selhub J, Paul L, Rosner B, et al. Prediagnostic leukocyte genomic DNA methylation and the risk of colorectal cancer in women. PLoS One. 2013;8(4):e59455.

21. Ally MS, Al-Ghnaniem R, Pufulete M. The relationship between gene-specific DNA methylation in leukocytes and normal colorectal mucosa in subjects with and without colorectal tumors. Cancer Epidemiol Biomark Prev. 2009; 18(3):922-8.

22. Gao HL, Wang X, Sun HR, Zhou JD, Lin SQ, Xing YH, et al. Methylation status of transcriptional modulatory genes associated with colorectal Cancer in Northeast China. Gut Liver. 2018;12(2):173-82.

23. Gao Y, Killian K, Zhang H, Yu K, Li QZ, Weinstein S, et al. Leukocyte DNA methylation and colorectal cancer among male smokers. World J Gastrointest Oncol. 2012;4(8):193-201.

24. Walters RJ, Williamson EJ, English DR, Young JP, Rosty C, Clendenning M, et al. Association between hypermethylation of DNA repetitive elements in white blood cell DNA and early-onset colorectal cancer. Epigenetics. 2013; 8(7):748-55

25. Cordero F, Ferrero G, Polidoro S, Fiorito G, Campanella G, Sacerdote C, et al. Differentially methylated microRNAs in prediagnostic samples of subjects who developed breast cancer in the European prospective investigation into nutrition and Cancer (EPIC-Italy) cohort. Carcinogenesis. 2015;36(10): 1144-53. 
26. Riboli E. The European Prospective Investigation into Cancer and Nutrition (EPIC): plans and progress. J Nutrit. 2001;131(1):170s-5s.

27. Maksimovic J, Phipson B, Oshlack A. A cross-package Bioconductor workflow for analysing methylation array data. F1000Res. 2016;5:1281.

28. Aryee MJ, Jaffe AE, Corrada-Bravo H, Ladd-Acosta C, Feinberg AP, Hansen $K D$, et al. Minfi: a flexible and comprehensive bioconductor package for the analysis of Infinium DNA methylation microarrays. Bioinformatics. 2014; 30(10):1363-9.

29. Fortin JP, Labbe A, Lemire M, Zanke BW, Hudson TJ, Fertig EJ, et al. Functional normalization of $450 \mathrm{k}$ methylation array data improves replication in large cancer studies. Genome Biol. 2014;15(11):503.

30. Chen YA, Lemire M, Choufani S, Butcher DT, Grafodatskaya D, Zanke BW, et al. Discovery of cross-reactive probes and polymorphic CpGs in the Illumina Infinium HumanMethylation450 microarray. Epigenetics. 2013;8(2):203-9.

31. Kolde R. Pheatmap: Pretty Heatmaps R Package Version 1.0.8. Available 711 online: https://CRAN.R-project.org/package=pheatmap. Accessed 4 Jan 2019

32. Phipson B, Maksimovic J, Oshlack A. missMethyl: an R package for analyzing data from Illumina's HumanMethylation450 platform. Bioinformatics. 2016; 32(2):286-8.

33. Du P, Zhang X, Huang CC, Jafari N, Kibbe WA, Hou L, et al. Comparison of Beta-value and $\mathrm{M}$-value methods for quantifying methylation levels by microarray analysis. BMC Bioinformatics. 2010;11:587.

34. Ritchie ME, Phipson B, Wu D, Hu Y, Law CW, Shi W, et al. limma powers differential expression analyses for RNA-sequencing and microarray studies. Nucleic Acids Res. 2015;43(7):e47-e.

35. Tibshirani R. Regression shrinkage and selection via the lasso. J Royal Statistical Soc, Series B (Methodological). 1996;58(1):267-88.

36. Venables WN, BD R. Modern applied statistics with S. 4th ed. New York: Springer; 2002.

37. Friedman J, Hastie T, Tibshirani R. Regularization paths for generalized linear models via coordinate descent. J Stat Softw. 2010;33(1):1-22.

38. Robin X, Turck N, Hainard A, Tiberti N, Lisacek F, Sanchez JC, et al. pROC: an open-source package for R and $\mathrm{S}+$ to analyze and compare ROC curves. BMC Bioinformatics. 2011;12:77.

39. Cho YA, Lee J, Oh JH, Chang HJ, Sohn DK, Shin A, et al. Genetic risk score, combined lifestyle factors and risk of colorectal Cancer. Cancer Res Treat. 2019;51(3):1033-40.

40. Jung KJ, Won D, Jeon C, Kim S, Kim TI, Jee SH, et al. A colorectal cancer prediction model using traditional and genetic risk scores in Koreans. BMC Genet. 2015;16:49.

41. Schmit SL, Edlund CK, Schumacher FR, Gong J, Harrison TA, Huyghe JR, et al. Novel common genetic susceptibility loci for colorectal Cancer. J Natl Cancer Inst. 2019;111(2):146-57.

42. Wang F, Dai CQ, Zhang LR, Bing C, Qin J, Liu YF. Downregulation of Lgr6 inhibits proliferation and invasion and increases apoptosis in human colorectal cancer. Int J Mol Med. 2018;42(1):625-32.

43. Thummuri D, Kumar S, Surapaneni SK, Tikoo K. Epigenetic regulation of protein tyrosine phosphatase PTPN12 in triple-negative breast cancer. Life Sci. 2015;130:73-80.

44. Alonso A, Sasin J, Bottini N, Friedberg I, Friedberg I, Osterman A, et al. Protein tyrosine phosphatases in the human genome. Cell. 2004;117(6):699-711.

45. Espejo R, Rengifo-Cam W, Schaller MD, Evers BM, Sastry SK. PTP-PEST controls motility, adherens junction assembly, and rho GTPase activity in colon cancer cells. Am J Physiol Cell Physiol. 2010;299(2):C454-63.

46. de Voer RM, Hahn MM, Weren RD, Mensenkamp AR, Gilissen C, van ZelstStams WA, et al. Identification of novel candidate genes for early-onset colorectal Cancer susceptibility. PLoS Genet. 2016;12(2):e1005880.

47. Li WH, Zhou ZJ, Huang TH, Guo K, Chen W, Wang Y, et al. Detection of OSR2, VAV3, and PPFIA3 methylation in the serum of patients with gastric Cancer. Dis Markers. 2016;2016:5780538.

48. Zhu L, Yan F, Wang Z, Dong H, Bian C, Wang T, et al. Genome-wide DNA methylation profiling of primary colorectal laterally spreading tumors identifies disease-specific epimutations on common pathways. Int J Cancer. 2018;143(10):2488-98.

49. Zhang YL, Wang RC, Cheng K, Ring BZ, Su L. Roles of Rap1 signaling in tumor cell migration and invasion. Cancer Biol Med. 2017;14(1):90-9.

\section{Publisher's Note}

Springer Nature remains neutral with regard to jurisdictional claims in published maps and institutional affiliations.

\section{Ready to submit your research? Choose BMC and benefit from}

- fast, convenient online submission

- thorough peer review by experienced researchers in your field

- rapid publication on acceptance

- support for research data, including large and complex data types

- gold Open Access which fosters wider collaboration and increased citations

- maximum visibility for your research: over $100 \mathrm{M}$ website views per year

At BMC, research is always in progress.

Learn more biomedcentral.com/submissions 